\title{
3D Simulations of Deep Directional Electromagnetic Tools in High-angle and Horizontal Wells
}

\author{
Vladimir Puzyrev (BSC) and Carlos Torres-Verdin (UT Austin)
}

78th EAGE Conference and Exhibition 2016

\section{Introduction}

With hydrocarbon exploration and production moving into areas of increasing geological and fluid complexity, the industry requires adoption of new technologies to enhance the efficiency of well placement. Electrical resistivity plays an important role in the characterization of geological formations due of its sensitivity to both porosity and hydrocarbon saturation. Given the limitations of the current electromagnetic (EM) logging-while-drilling (LWD) technology, new techniques such as very deep directional EM imaging have been introduced very recently (Seydoux et al., 2014). A new generation of directional imaging devices called mapping-while-drilling (MWD) tools includes the Schlumberger GeoSphere reservoir service (Ezioba and Denichou, 2014; Cogswell et al., 2014) which is claimed to be able to reveal subsurface bedding and fluid-contact details more than $30 \mathrm{~m}$ from the wellbore. This exceeds several times the formation coverage of conventional LWD technologies and provides an unprecedented depth of characterization, enabling operators to optimize well trajectory using real-time information, reduce drilling risk, maximize reservoir exposure, and increase production potential. Such benefits are, in particular, of great importance for high-angle (HA) and horizontal (HZ) wells that are commonly used nowadays to enhance hydrocarbon production (Ijasan et al., 2014). In this paper we invoke 3D forward simulation for modeling-based interpretation of deep directional EM responses in $\mathrm{HA} / \mathrm{HZ}$ wells and show the results of independent feasibility studies. The sensitivity of different components of the measured magnetic field is studied in detail for various frequencies and source-receiver offsets. Our workflow incorporates a parallel forward solver tailored for multi-source and multi-frequency problems.

\section{Modeling Cases}

We perform simulations for two main application areas illustrated in Fig. 1. The first one, referred to as the production or monitoring case, is to define the oil-water contact (OWC) which is the primary goal of determining the thickness of an oil column and estimating the well's production potential. This allows increasing the field recovery factor, maximizing production and diminishing the chance of early water breakthrough. The second case is the exploration problem when we aim at mapping resistive bodies (possible hydrocarbon reservoirs) in low-resistivity formations and shales in the vicinity of the wellbore. Such mapping can be used to find good-quality reservoir sands and to drill on target when performed in real time. The GeoSphere service has been reported to successfully map remote sand bodies at distances of 30-35 m from the wellbore (Ezioba and Denichou, 2014).
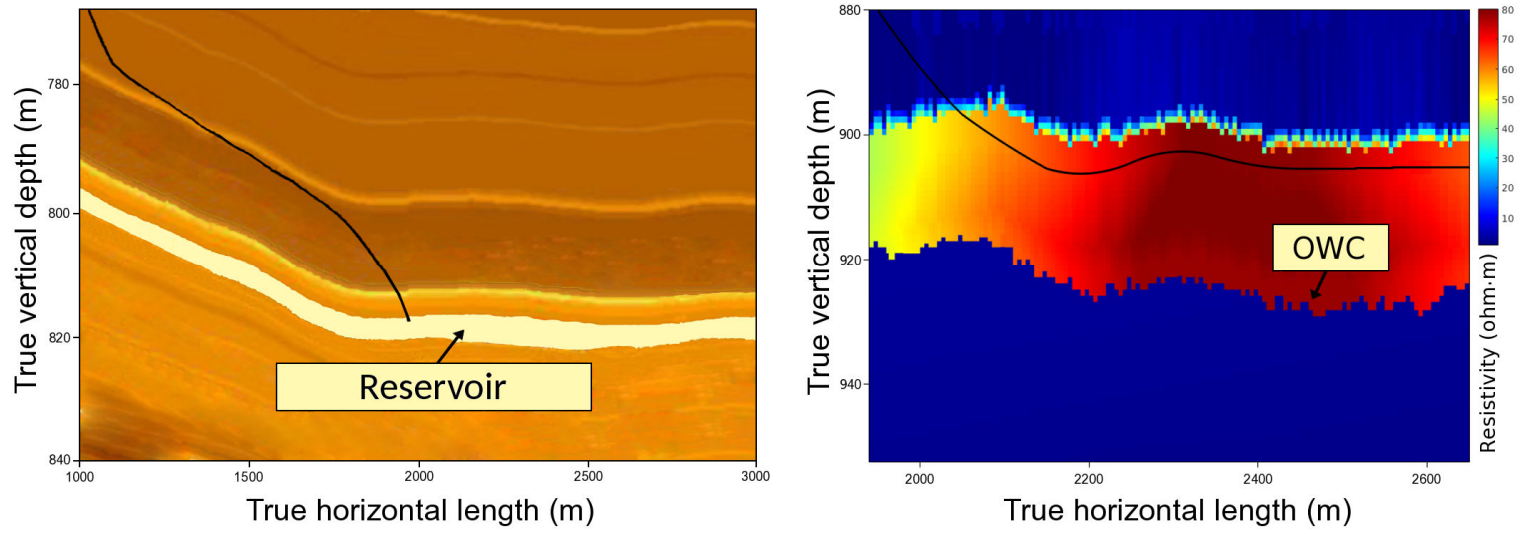

Figure 1: Choosing an optimal well trajectory after the top and bottom of the reservoir have been detected (left); model example in a horizontal development well (right).

Very deep directional techniques require multi-spacing and multi-frequency measurements and are no longer restricted to the detection of a single boundary based on simplified inversions or other methods. The interpretation of complex multilayered structures must rely on 3D modeling and 
inversion. However, despite that several 3D methods have been developed for the simulation of borehole EM measurements (e.g. Davydycheva et al., 2003; Hou et al., 2006), they have been often considered impractical for many applications due to the high computational cost of inversion. Recent progress in parallel computing has allowed us to develop an algorithm for accurate and efficient $3 \mathrm{D}$ simulations of multicomponent triaxial induction measurements in complex reservoir formations with extreme resistivity contrasts. The algorithm is based on the 3D forward modeling code of Puzyrev et al. (2015) that was modified to incorporate a new source type and a problem-specific linear solver. Results obtained with simulations for layered models were compared to those obtained with a 1D code and found to be in good agreement. This modeling algorithm allows one to precisely map the top and bottom of the reservoir and to delineate multiple subsurface layers, which is a first step toward full 3D inversion.

\section{Monitoring Examples}

In this subsection we examine the sensitivity of MWD technology to determining the OWC (i.e. the resistive/conductive contrast under the wellbore path). Multicomponent triaxial induction instruments are now routinely used in the industry for evaluation of resistivity anisotropy and formation dip. A set of these measurements includes nine orthogonal magnetic field components (three field components measured for each of the three mutually orthogonal transmitter coils). One of the goals of our study is to investigate which combinations exhibit high sensitivity to different model examples. Modeling at induction frequencies is of special interest because many commercial instruments operate in this frequency range.

For the first modeling test, we consider a simple model representing a production case. A horizontal well is located in a horizontal hydrocarbon reservoir. The top of the reservoir is located at $6 \mathrm{~m}$ true vertical depth (TVD) above the well path while the OWC is $20 \mathrm{~m}$ TVD below. We wish to estimate the effect that a non-flat OWC would cause on 3D simulation results. For this purpose, $10 \mathrm{~m}$ away from the magnetic source position we place a rectangular body with the same resistivity as the waterfilled rock below it. According to Archie's equation, the resistivity of a fluid saturated rock is related to its porosity, $\phi$, brine resistivity, $R_{w}$, and water saturation, $S_{w}$, as $R_{t}=a \cdot R_{w} \cdot \phi^{-m} \cdot S_{w}^{-n}$. The following rock/fluid properties and Archie's parameters are chosen in the modeling example: $\phi=0.25, R_{w}=0.13 \mathrm{Ohm}-\mathrm{m}, S_{w}=0.1$, the porosity exponent $m$ and the saturation exponent $n$ are chosen as 2, and Winsauer's factor $a$ is 1 .
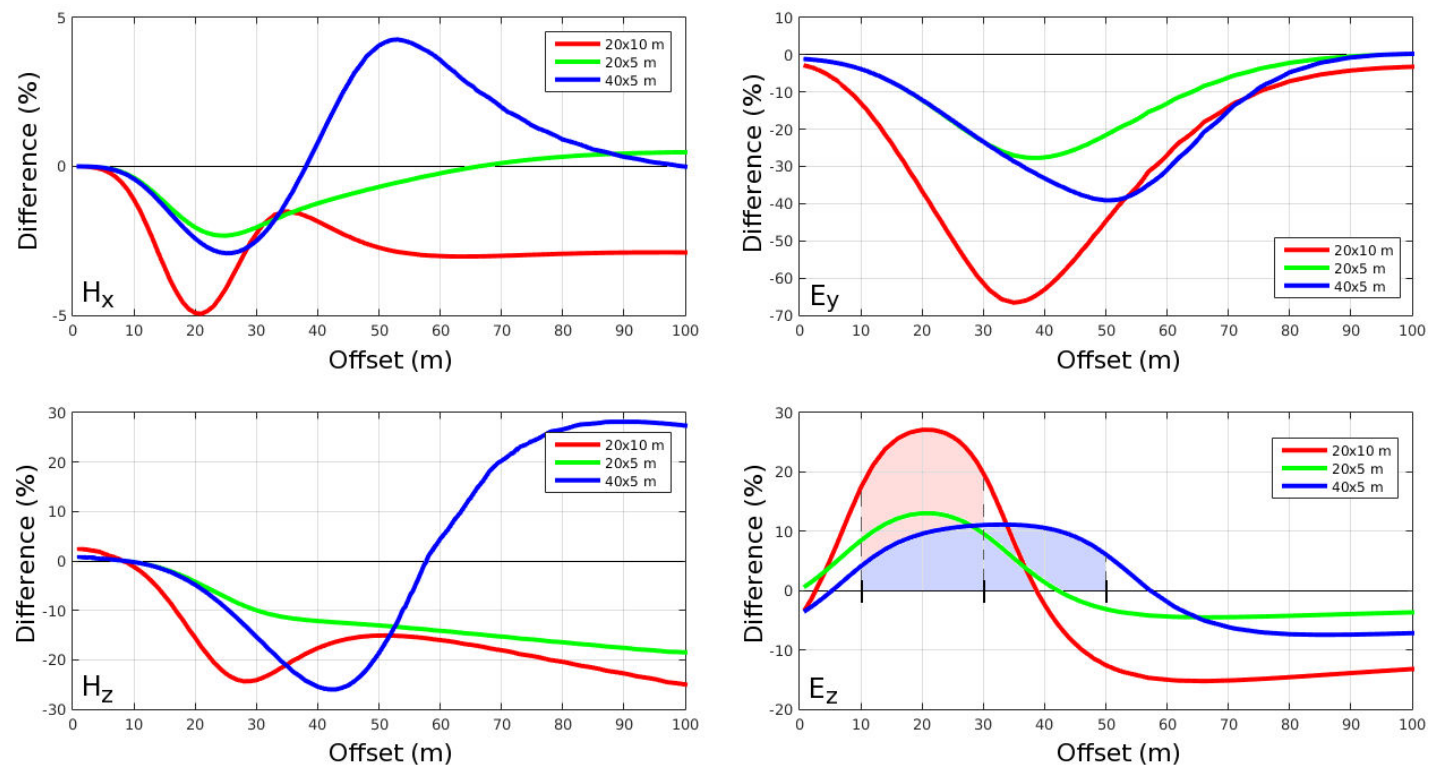

Figure 2: Relative changes in the amplitudes $\left(A-A_{r}\right) / A_{r}$ of the magnetic (left) and electric (right) fields for different anomaly sizes at $25 \mathrm{kHz}$. The source is a magnetic dipole oriented in $x$-direction. 
In Fig. 2, we show how variations in width and thickness of the obstacle affect the response of a triaxial induction instrument. Because the EM field amplitudes vary over several orders of magnitude, we plot the resulting fields normalized by the response of a layered model when the body is absent. Magnetic field components are of primary interest, however electric ones are shown as well given that they illustrate very clearly the change in the measurements in relation to the anomaly dimensions (see $E_{z}$ component in Fig. 2). We observe that the response of the model with the body can be stronger or weaker than the original one depending on the interaction with the reflections from the top and bottom of the reservoir. Results from the simulations at lower and higher frequencies are consistent with those shown in Fig. 2. Optimal transmitter offsets, as expected, depend on frequency and distance to the OWC. Fig. 3 illustrates the effect of moving the induction instrument above the anomaly. The strongest response is observed at large offsets $(50-60 \mathrm{~m})$ when the source is located directly above the anomaly. An offset of $30 \mathrm{~m}$ also brings useful information which will be complementary to the data measured with largest offsets. Two receivers located 30 and $60 \mathrm{~m}$ from the source would allow maximizing the response from the anomaly.
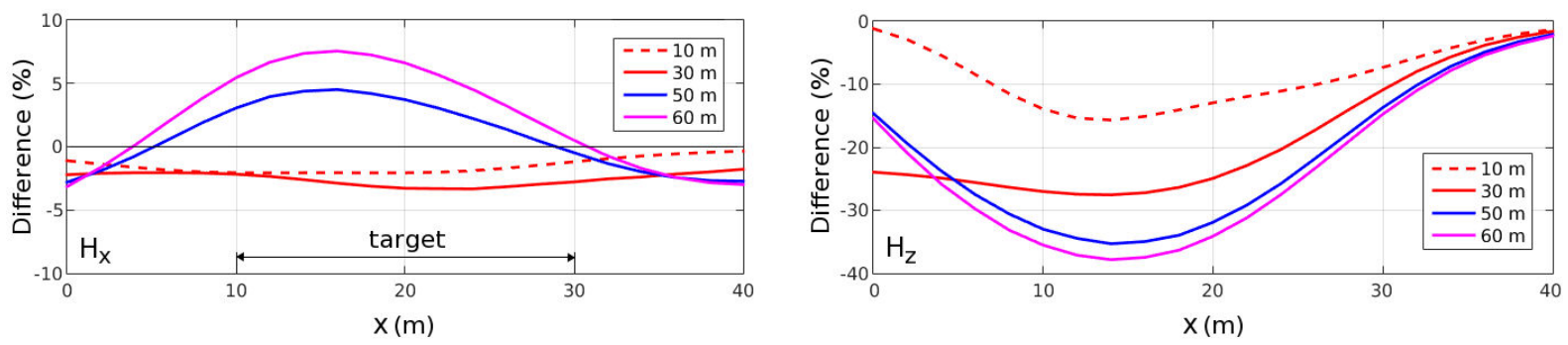

Figure 3: Relative changes in the amplitudes of the horizontal inline (left) and vertical (right) magnetic field components for different source positions and receiver offsets $(25 \mathrm{kHz})$.

\section{Exploration Example}

Fig. 4 shows the response of the MWD tool applied to locating sand targets. In this modeling situation, a small thin resistive body $(100 \Omega \cdot \mathrm{m})$ is located within the embedding shales of varying resistivity (1-5 $\Omega \cdot \mathrm{m})$ below the wellbore. The dimensions of the target are 20x20x5 $\mathrm{m}$ and its depth of burial varies from 10 to $20 \mathrm{~m}$. For the strongest $H_{x x}$ component, different responses are not clearly distinguishable in the amplitude-versus-offset plots, whereby the relative difference is marked additionally in Fig. 4. The target buried at $10 \mathrm{~m}$ depth produces a strong response, while the same body located twice as deep causes a maximum relative difference around $3 \%$ and $10 \%$ at 5 and 25 $\mathrm{kHz}$, respectively. At lower frequencies, the skin depth becomes much larger than the dimensions of the anomaly, while at higher ones the signal falls below the noise floor with the longest offsets.

Our modeling results confirm that deep directional EM tools are sensitive to small targets located a few tens of meters away from the well trajectory. Use of multiple transmitter-receiver offsets and frequencies in a wide band allows targeting different depths of investigation. Results of additional tests show that changes in shape and resistivity of the target are also clearly revealed by the change in magnetic fields measured at the receivers. However, the source-receiver configuration and modeling frequencies should be carefully selected because many of the combinations result in a relative change smaller than $3-5 \%$ which can be easily hidden in the signal noise.

\section{Conclusions and Future Directions}

We described results of full 3D modeling of a deep directional MWD instrument that allows the exploration and monitoring in near-wellbore zones at extended depths of investigation. Optimal configurations for several induction frequencies have been determined for various application conditions. A new inversion-based interpretation workflow is currently being developed using this 3D forward modeling algorithm. The combination of electromagnetic measurements acquired with new 
MWD tools and seismic amplitude measurements will make possible the optimal steering of wells in a geologically complex reservoir.
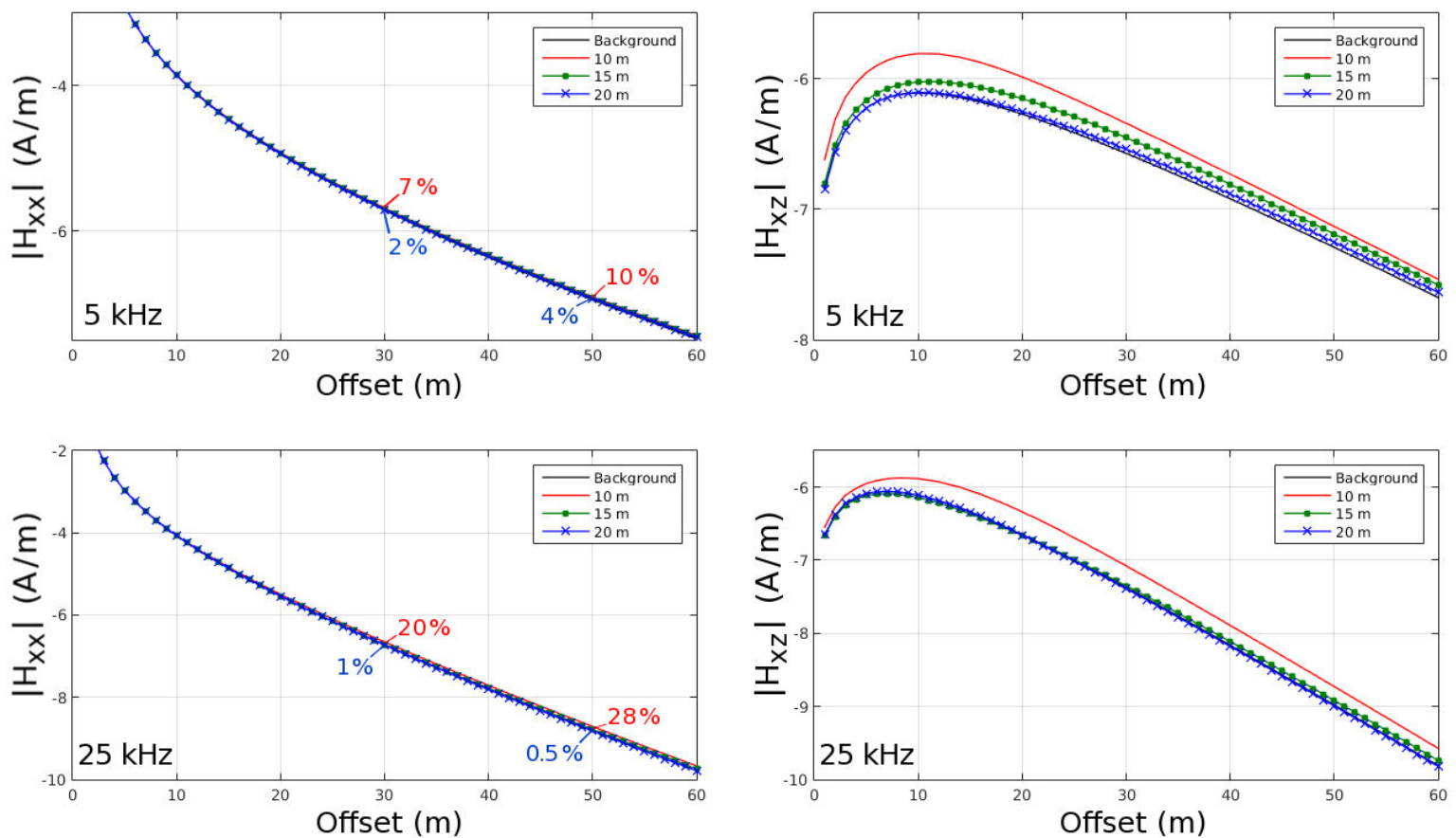

Figure 4: Amplitudes of the inline (left) and vertical (right) magnetic field components at $5 \mathrm{kHz}$ (top) and 25 $\mathrm{kHz}$ (bottom).

\section{Acknowledgments}

The first author acknowledges the RISE Horizon 2020 European Project GEAGAM (644202) for the travel support and the Repsol-BSC Research Center for funding. All numerical tests were performed on the MareNostrum supercomputer of the Barcelona Supercomputing Center. We also acknowledge the University of Texas at Austin's Research Consortium on Formation Evaluation.

\section{References}

Cogswell, T.L., Egan, M.J. and Kim, V. [2014] Application of GeoSphere logging tool for well placement and reservoir surveillance at Chayvo field, Sakhalin, Russia. Third EAGE International Science and Applications workshop Far East Hydrocarbons 2014, EAGE.

Davydycheva, S., Druskin, V. and Habashy, T. [2003] An efficient finite-difference scheme for electromagnetic logging in 3D anisotropic inhomogeneous media. Geophysics 68(5), 1525-1536.

Ezioba, U. and Denichou J.-M. [2014], Mapping-While-Drilling system improves well placement and field development. Journal of Petroleum Technology 66(08), 32-35.

Hou, J., Mallan, R.K. and Torres-Verdín, C. [2006] Finite-difference simulation of borehole EM measurements in 3D anisotropic media using coupled scalar-vector potentials. Geophysics 71(5), G225-G233.

Ijasan, O., Torres-Verdín, C., Preeg, W.E., Rasmus, J. and Stockhausen, E.J. [2014] Inversion-based interpretation of LWD resistivity and nuclear measurements: field examples of application in high-angle and horizontal wells. SPWLA 55th Annual Logging Symposium, Society of Petrophysicists and Well-Log Analysts.

Puzyrev, V., Gutierrez, N., Rodriguez, J.E., Hanzich, M. and de la Puente, J. [2015] Electromagnetic modeling using a massively parallel software framework. 77th EAGE Conference \& Exhibition, EAGE.

Seydoux, J., Legendre, E., Mirto, E., Dupuis, C., Denichou, J.-M., Bennett, N., Kutiev, G., Kuchenbecker, M., Morriss, C. and Yang, L. [2014] Full 3D deep directional resistivity measurements optimize well placement and provide reservoir-scale imaging while drilling. SPWLA 55th Annual Logging Symposium, Society of Petrophysicists and Well-Log Analysts. 